\title{
Isothermic Adsorption and Applications of Kinetics Models, Thermodynamics Functions ,Probability Sticking for Benzoic Acid and Some Substituted by Using Anew Adsorbent Substance
}

\author{
Khaleel-Ibrahim Al-Neimi Ahmed Ghanem Ahmed \\ Department of Chemistry, College of Education for pure science, University of Mosul \\ alnuaimi5310@yahoo.com
}

$\begin{array}{cc}\text { Received } & \text { Accepted } \\ \text { 03/03/2014 } & 08 / 05 / 2014\end{array}$

\begin{abstract}
The research including used anew adsorbent substance collected from sandstorms which arrived to Mosul city to study adsorption of Benzoic acid and some substituted and application of kinetics Models (pseudo first and second order reaction ) Elovich model kinetics and determination of thermodynamic function for adsorption process as well as the probability sticking for acids molecules on surface $\left(\mathrm{S}^{*}\right)$ and the appareant activation energy, the isothermic constants were determined for frendlich ,Langmir,Tempkin at different temperature and factors affecting on adsorption process ,the results gives indicates that the physical mechanism which predominate and some times physical and chemical the adsorption was increased and desorption decreased ,this process non spotonous and need lower energy to happened
\end{abstract}

Key wods: Carboxylic acid,adsorption , isotherm,New Clay,kinetic 


\title{
الأمتزاز الأيزوثيرمي وتطبيق النماذج الحركية والدوال الثرموداينيميكية واحتمالية الاتصاق
}

لحامض البنزويك وبعض معوضاته باستخذام مادة مازة جديدة

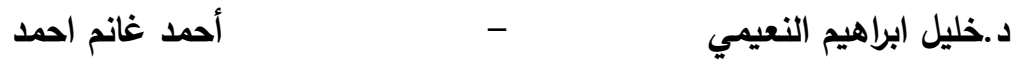 \\ قسم الكيمياء - كلية التربية - جامعة الموصل \\ تاريخ الاستلام ت ت إيخ القبول \\ 2014/05/08 2014/03/03
}

\begin{abstract}
الخلاصة
تضمن البحث استخام مادة مازة جديدة تم جمعها من العواصف الرملية القادمة الى مدينة الموصل

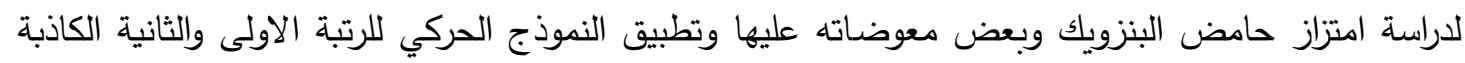

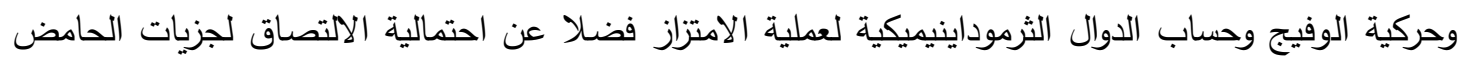

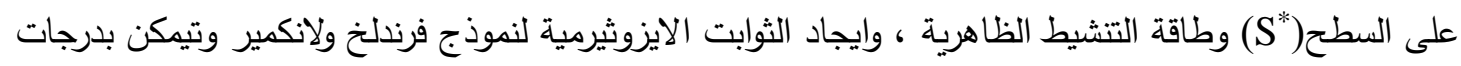

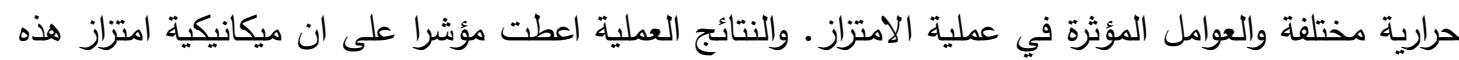

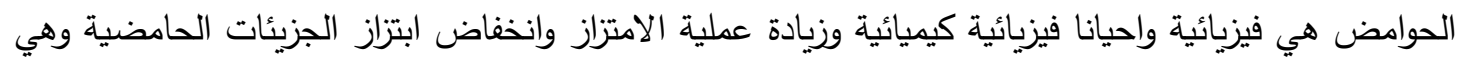
عملية غير تلقائية وتحتاج الى القليل من الطاقة لحدوثها.
\end{abstract}

الكلمات المفتاحية :حامض كاربوكسيلي،امتزاز،ايزوثيرم ,طين جديد,حركية 


\section{المقدمة}

يعتبر حامض البنزويك ومعوضاته مواد ملوثة كونها تستخدم في الصناعات الغذائية والدوائية وحقل الصناعة

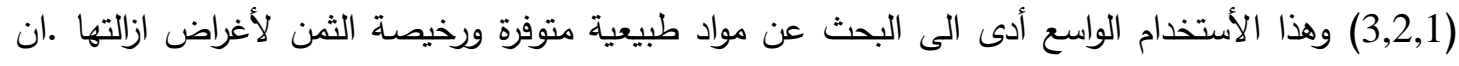

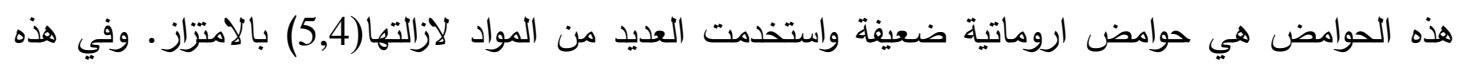
الدراسة استخدم الرمل (clay) الذي يحتوي بتركيبته على العديد من الاكاسيد الفلزية ذات الكفاءة العالية حيث

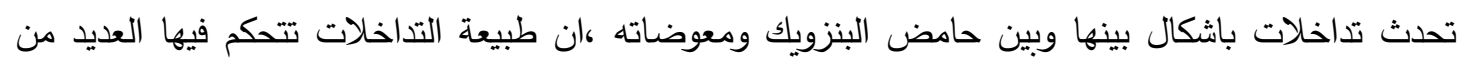
العوامل مثل نوع المجاميع المستقطبة الموجودة على السطح والتي بدورها ستحدد كون عملية الأمتزاز فيزيائية عن طريق التداخلات الكهروستاتيكية ،او تكون ذات طبيعة كيميائية ولكن في كل الأحوال تبدأ فيزيائية ،ان تغير التئي

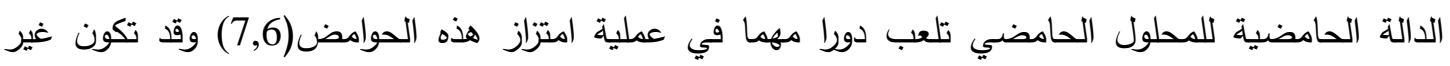

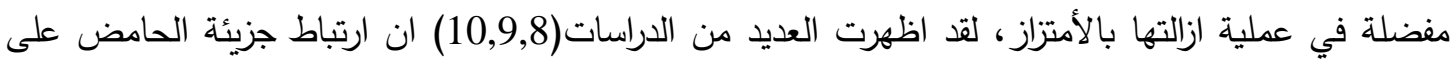

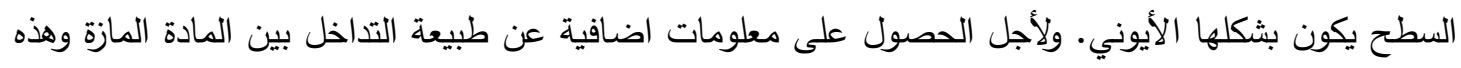

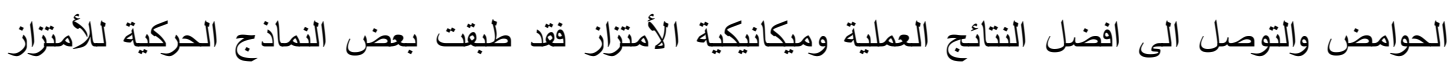

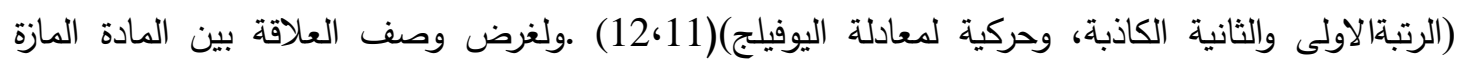

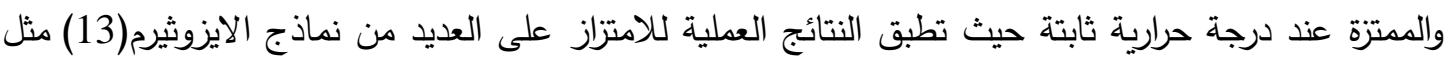

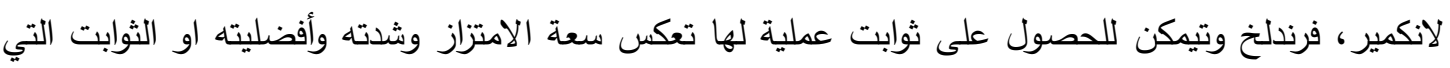

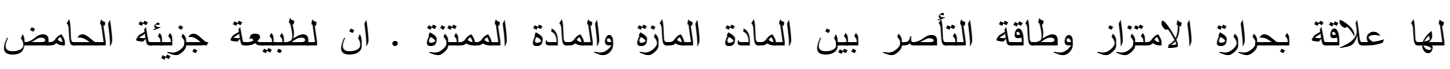
الكربوكسيلي القطبية واستقرارية الانيون بالريزوناس(14) ستؤثر كثيرا في عملية امتزازها من المحلول الى السطح

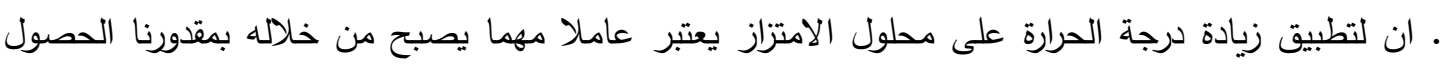
على الدوال الثرموداينيميكية لعملية الامتزاز والتي تعطي الصورة الواضحة عن طبيعة عملية الامتزاز .

\section{المواد وطرائق العمل}

1-المواد الكيمياوية:

ان المواد الكيمياويـة والمذيبات التي استخدمت في هذه الدراسـة مجهزة من شركتي(Fluka) و(BDH). وقد استخدم قسم من هذه المواد كما هي بعد التاكد من نقاوتها من خلال قياس درجة انصهارها. 2-المادة المازة المستخدمة في عملية الامتزاز

لقد جمعت مادة الرمل (clay) من العواصف الرملية القادمة الى مدينـة الموصل عن طريق تهيئة اواني دائرية بلاستيكية قطرها (60) سم نظيفة ومتعددة حيث يجمع الرمل الجاف من هذه الاواني مباشرة ويحفظ في حاويات

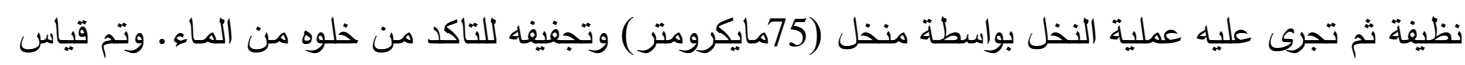

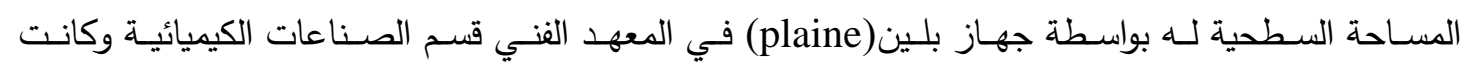
فضلا عن قياس مكوناته من قبل معمل سمنت بادوش باستخدام جهاز فلورة الاشعة السينية وكانت مكوناته حسب مامدرج في الجدول رقم (1). 
جدول (1)(المكونات الكيميائية للرمل (clay)

\begin{tabular}{|c|c|}
\hline $\mathrm{pH}$ & 8.87 \\
\hline $\mathrm{MgO}$ & 3.47 \\
\hline \hline $\mathrm{Al}_{2} \mathrm{O}_{3}$ & 5.91 \\
\hline \hline $\mathrm{SiO}_{2}$ & 19.71 \\
\hline $\mathrm{SO}_{3}$ & 0.48 \\
\hline \hline $\mathrm{CaO}$ & 37.36 \\
\hline \hline $\mathrm{Fe}_{2} \mathrm{O}_{3}$ & 1.89 \\
\hline
\end{tabular}

3 - الاجهزة المستخدمة:

Alectrothermal melting point 9300) قياس درجة الانصهار :- استخدم جهاز نوع) B - الهزاز ذي الحمام المائي:استخدم جهاز الهزاز المبرمج من نوع (Julabo sw23) والذي يحتوي بداخله على حمام مائي لغرض ضبط وتتظيم درجات الحرارة عند دراسة الامتزاز • C جهاز قياس الدالة الحامضية : استخدم جهاز DH meter من نوع (JEN WAY 3510) تم ضبطه ومعايرته باستخدام محاليل منظمة (pH9.pH4) وقياس فرق الجهد عند درجات حرارية (20،30،40،50، قبل عملية الامتزاز وبعدها. - فرن التجفيف:- جفف الرمل (clay) في فرن كهبائي للتاكد من خلوه من الماء.

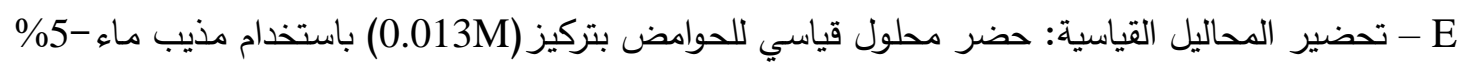
ايثانول بمقدار (100) مل لتحضير محاليل اقل تركيزا من الحامض واستخدم محلول هيدروكسيد الصوديوم الذي مئي تم ضبط تركيزه باستخدام محلول هيدروجين بوتاسيوم فثاليت الحامضية واستخدام دليل الفينولفثالين . دراسة العوامل المؤثرة في عملية الامتزاز:1 - دراسـة تاثير زمن التماس : درس زمن التماس بين السطح الصلب ومحلول الامتزاز لغرض تحديد زمن

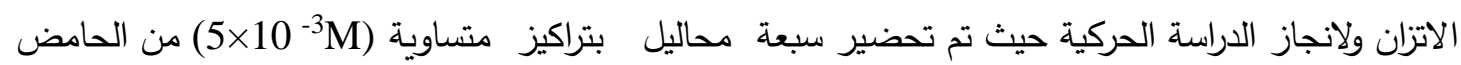

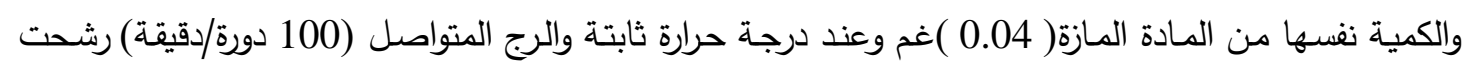

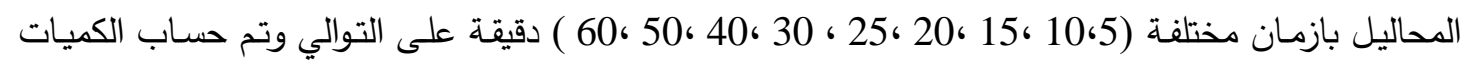

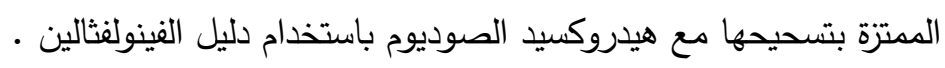


2 - تاثير التركيز الابتدائي :-

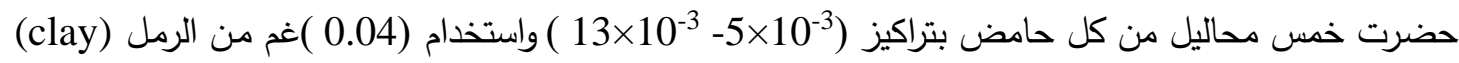
لكل محلول، رجت المحاليل لمدة (60 ) دقيقة على هزاز مبرمج ثم تم ترشيحها وحسبت كفاءة الامتزاز • 3- تحديد كمية المادة المازة :-

استخدم تركيز ثابت من المحلول ألحامضي (3010 5 5 ) مولاري وزمن تماس ثابت لكل حامض مع تغير كمية المادة المازة تراوحت بمدى (0.01 غم - 0.06 غم) للوصول بنظام الامتزاز الى حالة التوازن. 4 - تاثير الدالة الحامضية :-

بعد تعيين الظروف المثلى لعملية الامتزاز عند الدالة الحامضية الطبيعية لكل حامض درس ثاثير تغير الدالة

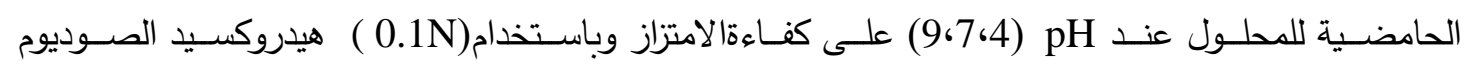
و) حامض الهيدروكلوريك لضبط الدالة الحامضية للمحاليل قبل اضـافة المادة المازة وباستخدام جهاز $\cdot(\mathrm{pH}$ meter $)$ 5- 20 - دراسة تاثير درجة الحرارة :تم تثبيت كافـة الظروف المثاليـة لعملية الامتزازوتم تغيير درجة الحرارة لمحاليل الحوامض ثم حسـاب كفاءة الامتزاز عن المدى الحراري المستخدم (20م-60م) وباستخدام حمام مائي مبرمج وقياس درجة حرائه درارة المحاليل

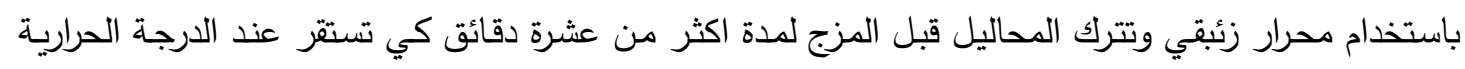
المطلوبة.

\section{دراسة حركية الامتزاز:-}

حضرت (50مل) من المحلول الحامضي بسبعة دوارق عند الظروف المثالية لامتزاز كل حامض وانجاز عملية

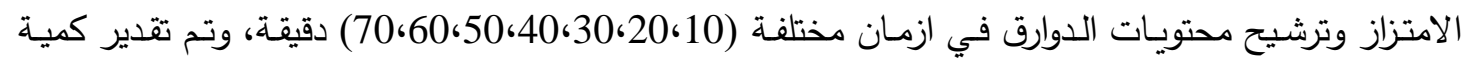
المادة الممتزة عند كل زمن وتم تطبيق النتائج العملية على النماذج الحركية المعرفة في الادبيات.

حساب الدوال الثرموداينيميكية:

تم اولا حساب ثابت توازن الامتزاز (K) عند درجات حرارية مختلفة عند حالة التوازن من النسبة بين تركيز المادة

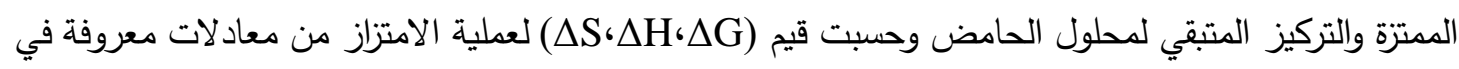
الادبيات .

\section{تطبيق ايزوثيرمات الامتزاز:-}

طبقت النتائج العملية للامتزاز على ايزوثيرم لانكمير ، فرندلخ وتيمكن لحساب ثوابتهما من خلال رسم العلاقات

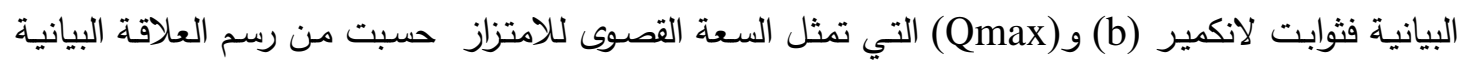

ضد (Ce/qe) 
سعة الامتزاز ملغم/غم qe

التركيز المتبقي ملغم/لتر

اما ثوابت فرندلخ فقد حسبت من معادلة فرندلخ وهي من رسم العلاقة البيانية بينlogq ضد المع. اما ثوابت تيمكن فقد حسبت من رسم العلاقة بين qu ضد lnCe والرسوم انجزت باستخدام برنامج (Excel) في الحاسوب.

\section{النتائج والمناقشة}

لقد استخدم الرمل (clay) الذي تحمله العواصف الرملية لاول مرة والهدف من ذلك هو معرفة الطبيعة التركيبية

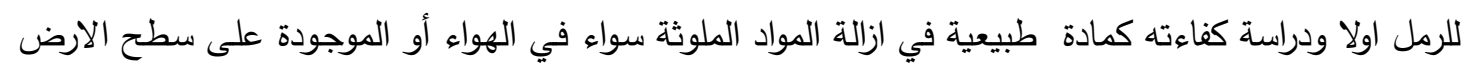
ثانيا وخصوصا بعد نزول المطر واختلاطه مع الرمل ليتحول شكل طين ومحلول رملي حيث اثبتت هذه الدئه الدراسة بثكل قطعي الكفاءة العالية في ازالة المركبات العضوية بوجود كميات صغيرة من الرمل (0.04)غم للحصول على نسب امتزاز عالية تصل الى اكثر من 90\%

1 - من التماس من النتائج العملية للامتزاز :

يعـد عامـل مهم فـي تقيـيم كفــاءة عمليـة الامتزاز حيـث تكـون سـريعة فـي (10-15) دقيقـة الاولـى مـن

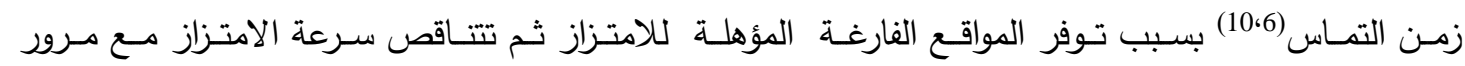

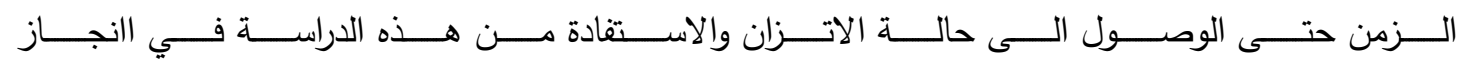

الدراسـة الحركية، حيث كان افضل زمن تماس لحامض البنزويك هو(30) دقيقة حيث الحصول على كفاءة امتزازمقدارها 82\% وتبقى ثابتة لحد (60) دقيقة ولمعوض (اورثو - هيدروكسي حامض البنزويك) كان زمن هون التماس بمقدار (40) دقيقة وتبقى الكفاءة ايضا ثابتة بعد هذا الزمن ونفس الحالة في معوض (بارا-هيدروكسي

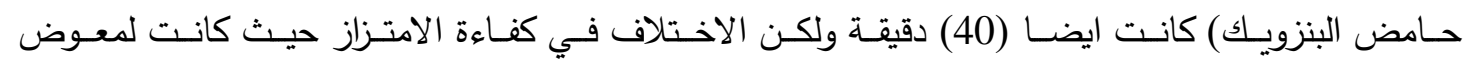
اورثو(90.2\%) بينما معوض بارا (74.0\%) اما الاختلاف في زمن تماس حامض البنزويك عن المعوضين هو بسبب وجود اصرة هيدروجينية ضمنية(15) في معوض اورثو وبينية في معوض بارا ، هذه التداخلات الجزيئية تؤثر في سرعة امتزاز الحامض على سطح الرمل فضلا عن تاثير الاعاقة الفراغية (15). 2- تاثير التركيز الابتدائي :-

يؤثر التركيز الابتدائي للمادة الممتزة تاثيرا كبيرا في كفاءة الامتزاز عندما يكون المتغير الوحيد في نظام الامتزاز

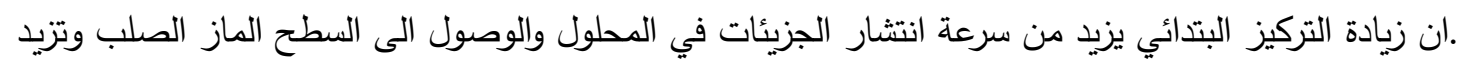

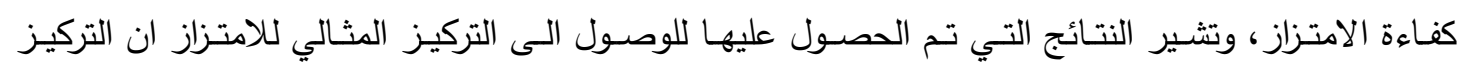

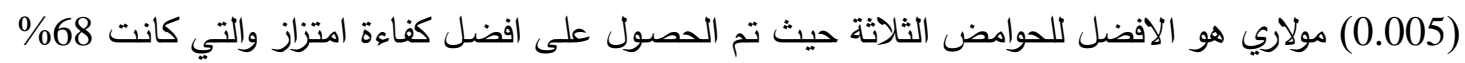
لحامض البنزويك، و75.5\% لمعوض اورثو و74\% لمعوض بارا ـ 
3- ت تاثير كمية المادة المازة :

تستخدم لتحديد سعة امتزاز المادة المازة لاية مادة ممتزة عند تركيز ابتدائي معلوم، ان زيادة المادة المازة يعني

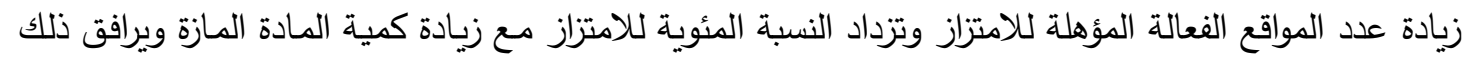
نقصان في سعة الامتزاز وقد كان افضل كمية مستخدمة هي (0.06)غم حيث كانت النسبة المئويـة للامتزاز لحامض البنزويك (97\%) ولمعوض اورثو(97.55\%) ولمعوض بارا (92\%).

4-تاثير الدالة الحامضية :-

توثر قيمة الدالة الحامضية للمحلول قي كفاءة عملية الامتزاز لتاثيرها في طبيعة المجاميع الفعالة الموجودة على

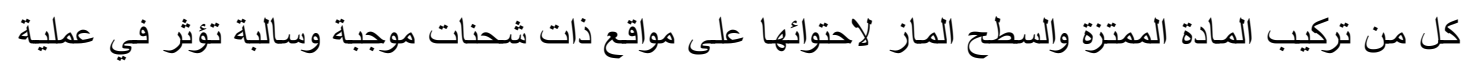

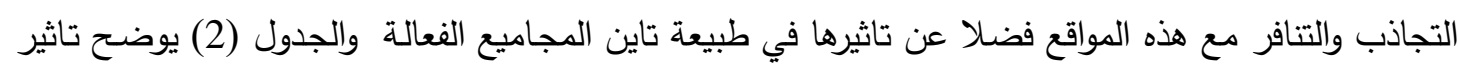

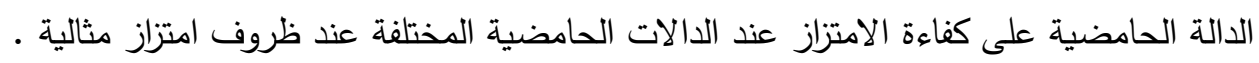

جدول رقم(2) تاثير pH على كفاءة الامتزاز عند الدالات الحامضية المختلفة عند ظروف امتزاز مثالية

\begin{tabular}{|c|c|c|c|}
\hline $\mathrm{pH}$ & \multicolumn{3}{|c|}{ \%Adsorption } \\
\hline & Benzoic acid & 0-hydroxy benzoic acid & p-hydroxy benzoic acid \\
\hline 4 & 72 & 95.2 & 76 \\
\hline Natural & 85.2 & 93.6 & 81.4 \\
\hline 7 & 99.0 & 99.2 & 94 \\
\hline 9 & 99.0 & 99.2 & 94 \\
\hline
\end{tabular}

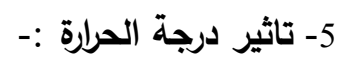

يتفاوت تاثير درجة الحرارة تبعا لنوع الامتزاز وطبيعته ففي حالة الامتزاز الفيزيائي فان زيادة درجة الحرارة تعمل على زيادة طاقة الجزيئة الحركية مما يؤدي الى فلك ارتباطها بالسطح وعودتها الى المحلول وهذا بدوره يؤدي الى لى لئي

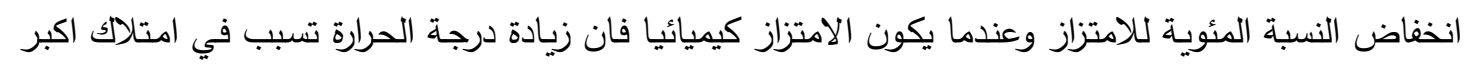
عدد من الجزيئات طاقة تتشيط كافية للارتباط بالسطح كيميائيا فتزداد كفاءة الامتزاز وتم تطبيق تغير درجة دئة

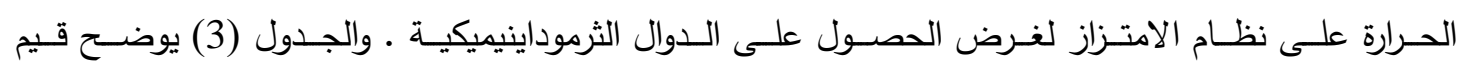
$\Delta \mathrm{S} ، \Delta \mathrm{H}$ ، $\Delta \mathrm{G}$ 
الجدول (3) يوضح قيم

\begin{tabular}{|c|c|c|c|c|}
\hline Carboxyli cacid & Temp.k & $\Delta \mathrm{G} \mathrm{kJ} / \mathrm{mol}$ & $\Delta \mathrm{H} \mathrm{KJ} / \mathrm{mol}$ & $\Delta \mathrm{S} \mathrm{J} / \mathrm{mol} . \mathrm{k}$ \\
\hline \multirow{5}{*}{ Benzoic acid } & 293 & -3.69 & \multirow{5}{*}{5.4273} & 31.13 \\
\hline & 303 & -3.96 & & 31.00 \\
\hline & 313 & -4.25 & & 30.93 \\
\hline & 323 & -4.64 & & 31.17 \\
\hline & 333 & -4.91 & & 31.05 \\
\hline \multirow{5}{*}{$\begin{array}{l}\text { o-hydroxy } \\
\text { benzoic acid }\end{array}$} & 293 & -5.35 & \multirow{5}{*}{11.1657} & 56.37 \\
\hline & 303 & -5.82 & & 56.08 \\
\hline & 313 & -6.35 & & 55.97 \\
\hline & 323 & -6.94 & & 56.07 \\
\hline & 333 & -7.61 & & 56.40 \\
\hline \multirow{5}{*}{$\begin{array}{l}\text { p-hydroxy } \\
\text { benzoic acid }\end{array}$} & 293 & -2.5478 & \multirow{5}{*}{8.4553} & 37.55 \\
\hline & 303 & -2.90 & & 37.4887 \\
\hline & 313 & -3.29 & & 37.53 \\
\hline & 323 & -3.72 & & 37.70 \\
\hline & 333 & -4.01 & & 37.44 \\
\hline
\end{tabular}

وتثير النتائج في الجدول(3) ان قليلة وتحمل اشـارة موجبة وهذا يدل على ان عملية الامتزاز غير

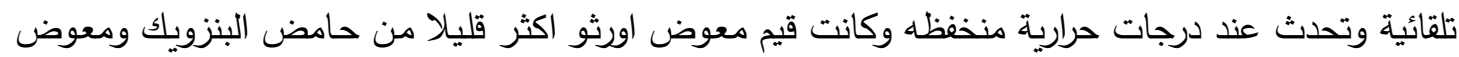
بارا بسبب احتواء الاول على اصرة هيدروجينية ضمنية بحلقة سداسية مستقرة. اما قيم $\Delta$ فهي ايضـا موجبة وهذا يشير الى وجود عملية امتصاص فضلا عن عملية الامتزاز حيث ان زيادة درجة الحرارة لحدود معينة تزيد من سرعة انتشار جزيئات الحامض على السطح وزيادة الفعل المتبادل بين السطح والجزيئة الممتزة وتظهر تلك الك

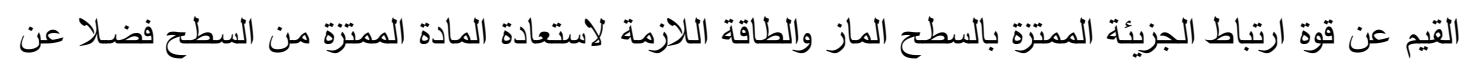

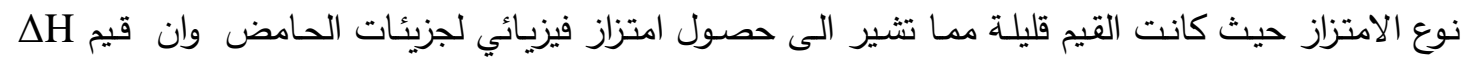
لمعوض اورثو اكبر من معوض بارا وهذا بدوره اكبرمن حامض البنزويك ونفس هذا التسلسل في قيم كانت قليلة ومتقاربة. 


\section{تطبيق بعض النماذج المركية على عملية الامتزاز:-}

ان حركيـة الامتزاز عمليـة معقدة تجري بمراحل متعددة بطاقـات تنشـيط مختلفـة تتضـن انتقـال الايونـات او الجزيئات من الطور السائل بعد التغلب على التداخلات الجزيئية وانتقالها الى السطح الصلب وان هذه الحركية

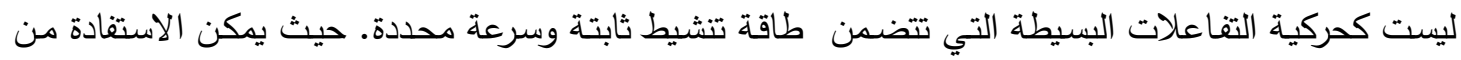
تاثيرزمن التماس بين المادة المازة والممتزة على سعة الامتزاز في فترات زمنية تتضمن الدقائق الاولى من عملية

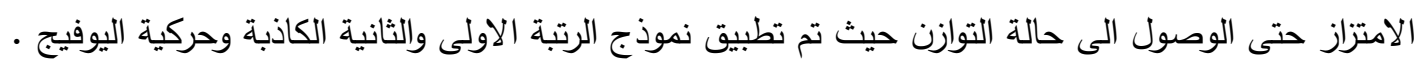

$\log \left(\mathrm{q}_{\mathrm{e}}-\mathrm{q}_{\mathrm{t}}\right)=\operatorname{loqq}_{\mathrm{e}}-\mathrm{k}_{1} / 2.303 \mathrm{t}$ المعادلة الحركية للرتبة الاولى الكاذبة :-

$$
\begin{aligned}
& \mathrm{t} / \mathrm{q}_{\mathrm{t}}=1 / \mathrm{k}_{2} \mathrm{q}_{\mathrm{e}}{ }^{2}+1 / \mathrm{q}_{\mathrm{e}} \mathrm{t} \\
& \mathrm{q}_{\mathrm{t}}=1 / \beta \ln (\alpha \beta)+1 / \beta \ln \mathrm{t}
\end{aligned}
$$$$
\text { المعادلة الحركية للرتبة الثانية الكاذبة :- }
$$$$
\text { معادلة النموذج الحركي لليوفيج :- }
$$

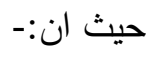

$$
\text { = qe }
$$

= سعة الامتزازعند ازمان مختلفة ملغم/غم. qt

$$
\text { Kابت سرعة الرتبة الاولى الكاذبة (min } \text { K }_{1}
$$

(g.mg-1.min'

( m.mole/g.min) السر عة الابتدائية للامتز از =a

$$
\text { ק= ثابت الابتزاز (g/m mole) }
$$

لقد طبقت الدراسة الحركية على الحوامض عند الظروف المثلى لعملية الامتزاز ،ان معرفة مدى انطباق النموذج

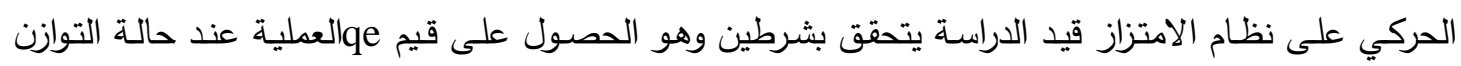

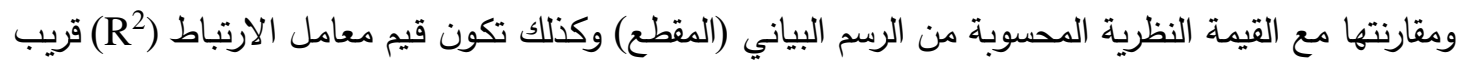

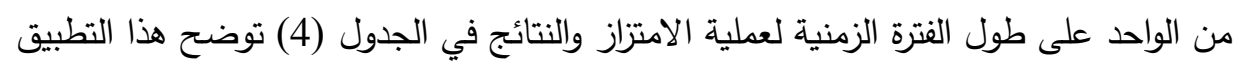


جدول(4) تطبيق نموذجي الرتبة الاولى والثانية الكاذبة على النتائج العملية للامتزاز لحامض

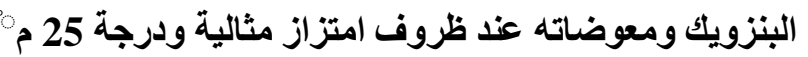

\begin{tabular}{|c|c|c|c|c|c|c|c|c|}
\hline \multirow{2}{*}{$\begin{array}{l}\text { carboxylic } \\
\text { acid }\end{array}$} & \multirow{2}{*}{$\begin{array}{c}\text { qe } \\
\text { experimental } \\
\mathrm{mg} / \mathrm{gm}\end{array}$} & \multicolumn{3}{|c|}{ First order Model } & \multicolumn{4}{|c|}{ second order Model } \\
\hline & & $\mathrm{K}_{1}(\min )^{-1}$ & $\begin{array}{c}\mathrm{q}_{\mathrm{e}} \text { theortical } \\
\mathrm{mg} / \mathrm{gm}\end{array}$ & $\mathrm{R}^{2}$ & $\begin{array}{c}\mathrm{K}_{2} \\
\text { g.mg } \mathrm{mg}^{-1} \cdot \mathrm{min}^{-1}\end{array}$ & qetheortical & $\mathrm{R}^{2}$ & $\begin{array}{c}\mathrm{h} \\
\mathrm{mg} \cdot \mathrm{gm}^{-1} \\
. \mathrm{min}^{-1} \\
\end{array}$ \\
\hline Benzoic acid & 625.86 & 0.117 & 313.24 & 0.992 & $33 \times 10^{-5}$ & 1000 & 0.999 & 333.3 \\
\hline $\begin{array}{c}\text { o-hydroxy } \\
\text { Benzoic acid }\end{array}$ & 778.65 & 0.087 & 280.05 & 0.984 & $5 \times 10^{-4}$ & 1000 & 0.998 & 500 \\
\hline $\begin{array}{c}\text { p-hydroxy } \\
\text { Benzoic acid }\end{array}$ & 638.80 & 0.101 & 322 & 0.984 & $25 \times 10^{-5}$ & 1000 & 0.999 & 250 \\
\hline
\end{tabular}

$$
\text { نلاحظ من النتائج في الجدول(4) مايلي:- }
$$

1-عند تطبيق نموذج الرتبة الاولى الكاذبة لوحظ اكتساب معوض اورثو هيدروكسي حامض البنزويك اعلى

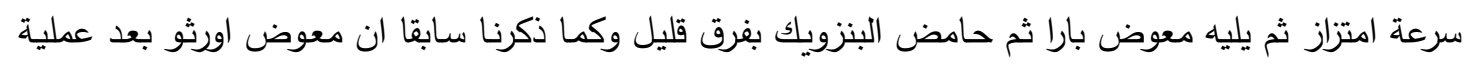

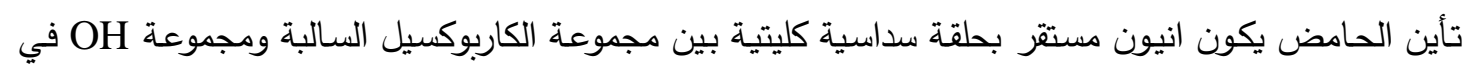
الموقع اورثو(15،15) وهذا يجعله اكثر سرعة في عملية الامتزاز كذلك فان قيم معامل الارتباط كانت ذات قيمة

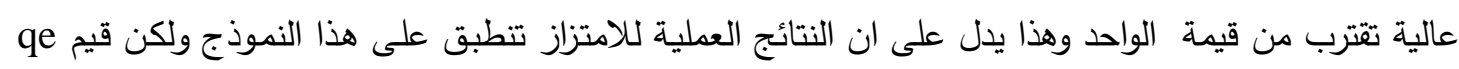

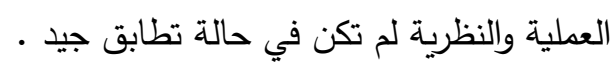

2-عند تطبيق نموذج الرتبة الثانية فان قيم ثابت سرعة الامتزاز (k2) اقل كثيرا من قيم (k1) للرتبة الاولى

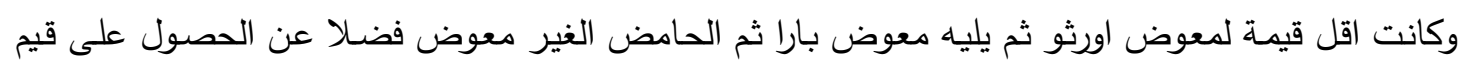

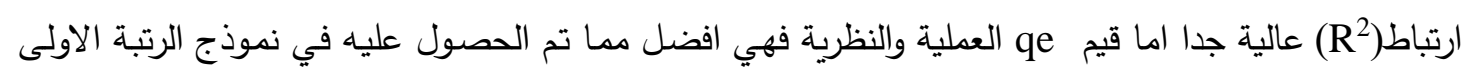

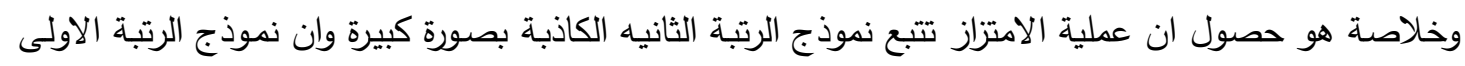

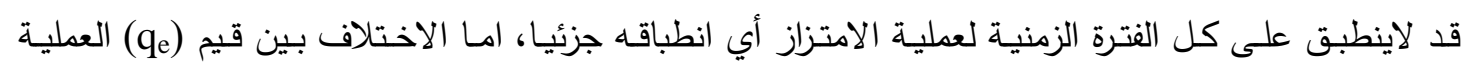

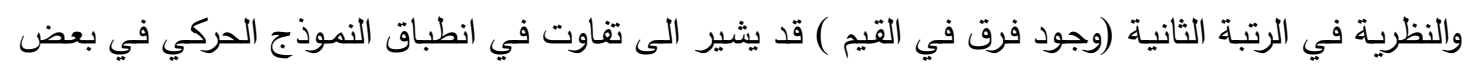

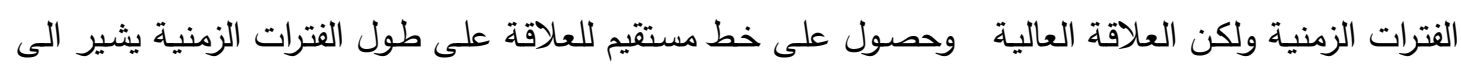

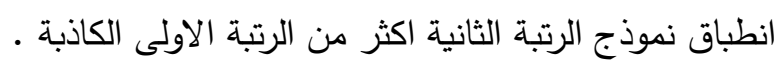

3- ان الملاحظة الجديرة بالاهتمام هو تاثير المجاميع المعوضـة على الحلقة الاروماتية للحامض على ثابت

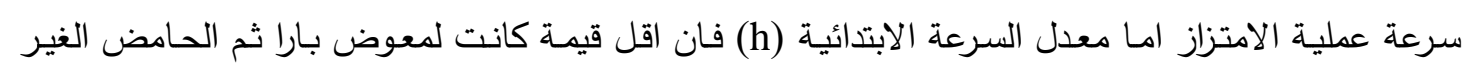

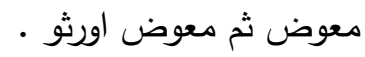




\section{تطبيق النموذج الحركي لليوفيج:-}

ان ثابتي حركية الامتزاز م،ه لليوفيج يتغيران كدالة للتركيز الابتدائي للمادة الممتزة فضلا عن تغيرهما كدالة

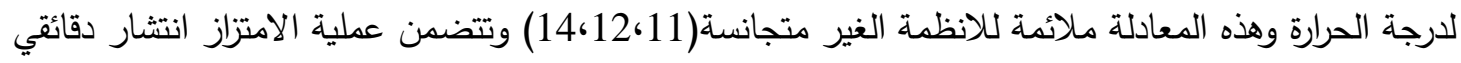
ضمني ( Intraparticle diffusion ) وان السرعة الابتدائية للانتشار الدقائقي الضمني تحسب من المعادلة

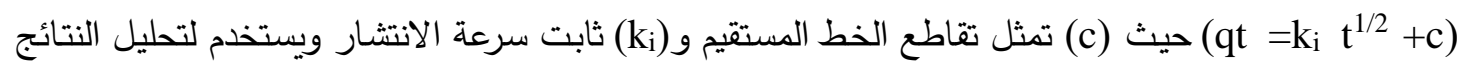

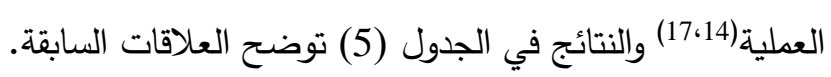

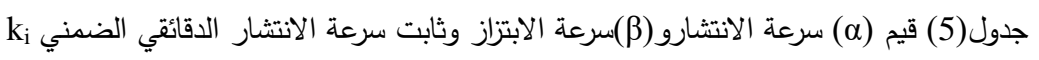

\begin{tabular}{|c|c|c|c|c|}
\hline $\begin{array}{c}\text { Carboxylic } \\
\text { acid }\end{array}$ & $\begin{array}{c}\alpha \\
\text { m.mol/g.min }\end{array}$ & g/m.mole & mg/g.min ${ }^{1 / 2}$ & $\mathrm{R}^{2}$ \\
\hline Benzoic acid & 2438 & 0.0105 & 50.43 & 0.994 \\
\hline $\begin{array}{c}\text { o-hydroxy } \\
\text { Benzoic acid }\end{array}$ & 17045 & 0.0114 & 42.36 & 0.975 \\
\hline $\begin{array}{c}\text { p-hydroxy } \\
\text { Benzoic acid }\end{array}$ & 1166.4 & 0.009 & 48.58 & 0.977 \\
\hline
\end{tabular}

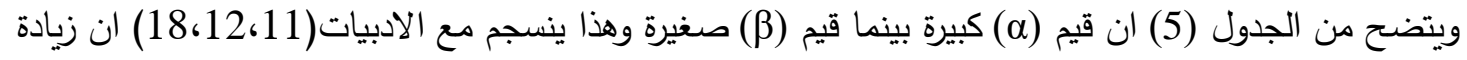

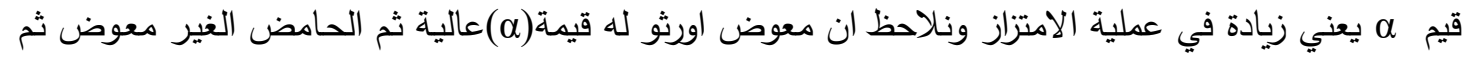

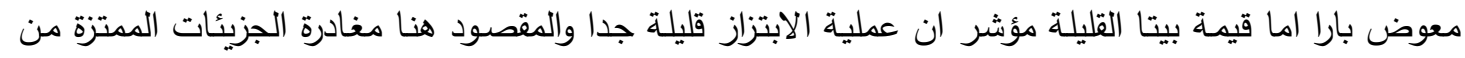
السطح الى المحلول وان عملية الابتزاز في معوض اورثو اكبر ثم الحامض الغير معوض ثم معوض بارا وهذا التسلسل ينسجم مع عملية الامتزاز ان تطبيق نموذج اليوفيج يسهل الكثف عن ميكانيكية الانتشار خلال عملية الانسية الامتزاز والملاحظ ايضا ان قيم qt التي تم الحصول عليها والتي حسبت منها النماذج الحركية ذات قيم عالية وهذايشير الى حصول ميكانيكية انتشار دقائقي ضمني جيد في عملية الامتزاز وتعتبر الخطوة المحددة لسرعة

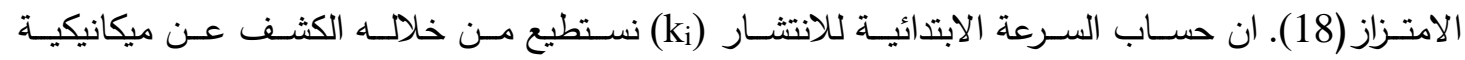

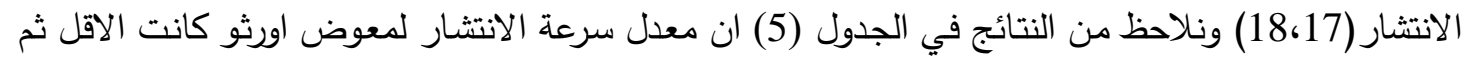
معوض بارا واعلى قيمة لحامض البنزويك وقد وضحنا سابقا ان وجود اصرة هيدروجينية ضمنية تثكل حلقة سداسية كليتية مستقرة ستؤدي الى تكوين حلقتين اروماتية وكليتية وهذا العامل يؤثر على سرعة انتشار جزيئة الحامض بتاثير الاعاقة الفراغية بينما في معوض بارا تكوين تداخلات جزيئية . 
حساب احتمالية الاتتصاق (S) وطاقة التنشيط الظاهرية:

ان الحصول على قيمة (S*) يعطي مؤشرجيد عن طبيعة الامتزاز من ناحية حدوثه وهل هو فيزيائي ام كيميائي او مزيج منهما وافضلهما عندما تكون قيمة (She التي تدعم الدراسة الثرموداينيميكية من ناحية حصول الامتزاز عند الدرجات (apparent activation energy) الحرارية المفضلة للدحلول حيث تستخدم معادلة خاصة مطورة عن معادلة ارهينوس وبالثكل الاتي:

$$
\mathrm{S}^{*}=(1-\Theta)-\mathrm{Ea} / \mathrm{RT}
$$

حيث $\Theta$ تمثل مقدار التغطية على السطح وتحسب عند درجات حراريـة مختلفة وترسم العلاقة البيانية بين

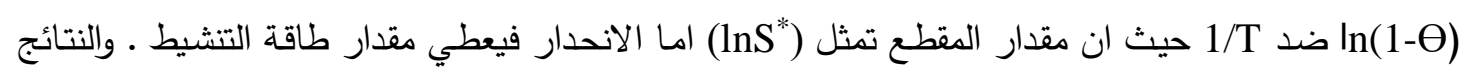

مدرجة في جدول رقم (6).

جدول (6) قيم("S) وطاقة التنشيط الظاهرية لحامض البنزويك وبعض معوضاته بتراكيز مختلفة وعند درجة حرارة 25مْ

\begin{tabular}{|c|c|c|c|c|c|c|c|c|c|}
\hline \multirow{2}{*}{ Conce(M) } & \multicolumn{3}{|c|}{ Benzoic acid } & \multicolumn{3}{c|}{ o-hydroxy benzoic acid } & \multicolumn{3}{c|}{ p- hydroxy benzoic acid } \\
\cline { 2 - 10 } & $\mathrm{S}^{*}$ & $\begin{array}{c}\text { Ea } \\
\text { KJ/mole }\end{array}$ & $\mathrm{R}^{2}$ & $\mathrm{~S}^{*}$ & Ea KJ/mol & $\mathrm{R}^{2}$ & $\mathrm{~S}^{*}$ & $\begin{array}{c}\text { Ea } \\
\mathrm{KJ} / \mathrm{mol}\end{array}$ & $\mathrm{R}^{2}$ \\
\hline 0.005 & 0.028 & -4.54 & 0.98 & 0.00149 & -10.28 & 0.98 & 0.0175 & -6.57 & 0.99 \\
\hline 0.007 & 0.1397 & -2.36 & 0.96 & 0.1742 & -1.445 & 0.99 & 0.2576 & -1.246 & 0.89 \\
\hline 0.009 & 0.1926 & -2.20 & 0.92 & 0.211 & -1.677 & 0.98 & 0.366 & -0.916 & 0.97 \\
\hline 0.011 & 0.2797 & -1.74 & 0.88 & 0.366 & -0.716 & 0.96 & 0.394 & -1.1340 & 0.96 \\
\hline 0.013 & 0.256 & -2.13 & 0.90 & 0.4690 & -0.5045 & 0.99 & 0.5096 & -0.663 & 0.97 \\
\hline
\end{tabular}

وعند التدقيق في نتائج الجدول نلاحظ الاتي:

1- ان قيم (S*) تزداد بزيسادة تركيز المحلول الحامضـي وهذا يشير الى ان الانتقال من الالتصـاق المفضل

بميكانيكيـة الامتزاز الفيزيائي الى علاقة امتزاز والتصـاق خطية بين المـادة المـازة والممتزة بميكانيكيـة امتزاز

فيزيائي وكيميائي (19).

2 ان طاقة التنشيط الظاهرية تحمل الاشارة السالبة وكما هو معروف علميا يجب ان تكون( Ea) موجبة ولكن هنا تمثل طاقة التتشيط الظاهريـة التي تعطي توضيح مفادة ان درجـة حرارة المحلول المنخفظة هي المفضلة لعملية الازالة بواسطة الامتزاز على السطح وان عملية الامتزاز هي عملية انتثار مسيطر عليها (18) والملاحظ 
ان طاقة التنشيط تقل بزيـادة التركيز أي توفر عدد كبير من الجزيئات يسـاعد على التنافس في الالتصـاق والامتزاز على السطح من دون الحاجة الى طاقة اضافية للمحلول الحامضي وتحول الامتزاز من حالة امتزاز فيزيائي الى مزيج من فيزيائي وكيميائي.

3-الملاحظ ان قيم (S) لمعوض بارا اكبر من حامض البنزويك ومعوض اورثو وهذا يشير ان حامض البنزويك وبعده معوض اورثو تحاول فيه الجزيئات الى الوصول الى حالة امتزاز تكون فيها ميكانيكية الامتزاز الكيميائي هي السائدة وأن ألالتصاق على السطح قيمها جدا عالية وفيزيائية بينما معوض بارا تسود فيه الانزياح

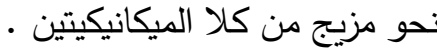
نتـائج تطبيق ايزوثيرمـات الامتزاز: لقد طبقت نمـاذج الايزوثيرم على الحوامض الثلاثة عند درجات حراريـة مختلفة وعند ظروف امتزاز مثالية والنتائج مدرجة في الجدول (7). 


\section{جدول(7) قيم ثوابت ايزوثيرم فرندلخ، لانكمير ، تيمكن عذد درجات حرارية مختلفة وظروف امتزاز مثالية}

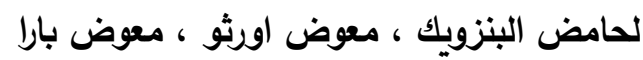

\begin{tabular}{|c|c|c|c|c|c|c|c|c|c|c|}
\hline \multirow[b]{2}{*}{ Temp c } & \multicolumn{3}{|c|}{ ايزوثيرم فرندلخ } & \multicolumn{4}{|c|}{ ايزوثيرم لانكمير } & \multicolumn{3}{|c|}{ ايزوثيرم تيمكن } \\
\hline & $\mathrm{K}_{\mathrm{f}}$ & $\mathrm{n}$ & $\mathrm{R}^{2}$ & $\begin{array}{l}\text { Qmax } \\
\text { mg/gm }\end{array}$ & $\mathrm{b}$ & $\mathrm{K}_{\mathrm{L}}$ & $\mathrm{R}^{2}$ & $\mathrm{~B}_{\mathrm{T}}$ & $\mathrm{K}_{\mathrm{T}}$ & $\mathrm{R}^{2}$ \\
\hline \multicolumn{11}{|c|}{ Benzoic acid } \\
\hline 20 & 404.5 & 11.11 & 0.91 & 1000 & 0.0169 & 16.9 & 0.99 & 62.68 & 172.97 & 0.89 \\
\hline 30 & 395.3 & 10.10 & 0.95 & 1000 & 0.0181 & 18.18 & 0.99 & 70.03 & 73.24 & 0.94 \\
\hline 40 & 403.6 & 10.20 & 0.96 & 1000 & 0.0192 & 19.2 & 0.99 & 70.53 & 80.95 & 0.95 \\
\hline 50 & 396.2 & 9.523 & 0.88 & 1000 & 0.0163 & 16.3 & 0.98 & 77.63 & 39.55 & 0.86 \\
\hline 60 & 356.45 & 7.692 & 0.91 & 1000 & 0.0140 & 14.08 & 0.98 & 98.41 & 7.02 & 0.88 \\
\hline \multicolumn{11}{|c|}{ o-hydroxy benzoic acid } \\
\hline 20 & 539.5 & 12.195 & 0.89 & 1000 & 0.0277 & 27.7 & 0.99 & 71.0 & 662.33 & 0.88 \\
\hline 30 & 557.18 & 12.650 & 0.88 & 1000 & 0.030 & 30 & 0.99 & 68.98 & 1131.18 & 0.86 \\
\hline 40 & 575.4 & 13.33 & 0.87 & 1000 & 0.033 & 33 & 0.99 & 66.68 & 2132.3 & 0.85 \\
\hline 50 & 599.7 & 14.28 & 0.85 & 1000 & 0.035 & 35.7 & 0.99 & 62.91 & 5578.13 & 0.85 \\
\hline 60 & 622.3 & 15.15 & 0.84 & 1000 & 0.04 & 40 & 0.99 & 59.65 & 14854.15 & 0.82 \\
\hline \multicolumn{11}{|c|}{ p-hydroxy benzoicacid } \\
\hline 20 & 442.5 & 13.8 & 0.89 & 1000 & 0.0212 & 21.27 & 0.998 & 49.68 & 2382.16 & 0.89 \\
\hline 30 & 461.3 & 14.49 & 0.93 & 1000 & 0.0208 & 20.8 & 0.998 & 48.59 & 4460.2 & 0.93 \\
\hline 40 & 487.5 & 16.12 & 0.95 & 1000 & 0.0222 & 22.2 & 0.998 & 45.08 & 45.08 & 0.94 \\
\hline 50 & 523.6 & 18.51 & 0.87 & 1000 & 0.025 & 25 & 0.998 & 39.41 & 39.41 & 0.86 \\
\hline 60 & 519.9 & 17.24 & 0.95 & 1000 & 0.0238 & 23.8 & 0.999 & 43.70 & 43.70 & 0.94 \\
\hline
\end{tabular}

نلاحظ من النتائج في الجدول (7) ان قيم ثوابت فرندلخ n، kf واللتان تعكسان سعة الامتزاز وشدته على التوالي

أي نسبة انتشار المادة الممتزة بين السطح والمحلول عند التوازن وتفضل قيمة (n)(عندما تكون قيمتها اكبر من

الواحد أي ان الامتزاز فيها فيزيائي وهو المفضل والنتائج تظهر ان نسبة انتشار المادة كبير ويزداد بزيادة درجة 
الحرارة اما قيم (kff فتشير الى افضلية للامتزاز ويكون فيزيائي وهذه القيم ايضـا تزداد بزيادة درجة الحرارة وان

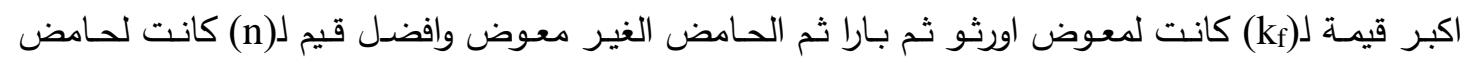
البنزويك وهي المفضلة ثم معوض اورثو ثم بارا، اماقيم ثوابت لانكمير (Qmax) التي تمثل اعلى سعة امتزاز فقد كانت متساوية لكل الحوامض وهي ذات قيمة ثابتة عند الدرجات الحرارية كافة ، في حين كانت قيم (b) التي تمثل قوة ارتباط الجزيئة الممتزة على السطح الماز فانها في حامض البنزويك تزداد بزيادة درجة الحرارة لحد 40خ ثم تنخفض وهذا يدعم حاجة نظام الامتزاز لدرجات حرارية قليلة لانجاز الامتزاز الفيزيائي وان زيادة درجة الحرارة عن هذه الحدود تؤدي ضـف قوة ارتباط الجزيئة بالسطح الماز بسبب زيادة الاهتزازات الجزيئية الناتجة من زيادة طاقتها وهذا يتسبب في فلك ارتباط الجزيئة وعودتها الى المحلول وهذايدل على ان نظام الامتزاز ذو طبيعة فيزياويـة والملاحظ من تطبيق النموذجين السـابقين ان تطابق النتائج العملية للامتزاز كانت جيدة مـع ايزوثيرم لانكمير للحصول على معامل ارتباط عالي جدا (R²=0.998) وهذا التطابق بنسب اقل لايزوثيرم فرندلخ وتيمكن . ان قيم (BT) التى لها علاقة بسعة الامتزاز تزداد بزيادة درجة الحرارة لحامض البنزويك في حين تنخفض في معوضي اورثو وبارا ،اما قيم (kT) التي لها علاقة بطاقة التاصر فانها تنخفض بزيادة درجة الحرارة لحامض البنزويك بينما تزداد في معوضسي اورثو وبارا وكانت قيم معامل الارتباط متفاوتة بين القيم الجيدة والواطئة والنتائج السابقة تتفق مع الادبيات.

\section{الاستنتاجات}

لقد تبين من خلال النتائج التي تم الحصول عليها كفاءة واهمية المادة المازة الجديدة وهو الطين الذي تم جمعه من العواصف الرملية القادمة الى مدينة الموصل وان سبب ارتفاع كفاءة امتزازه هو احتواءه على اكاسيد فلزية متعددة أي ان عدد المواقع المتاحة للامتزاز اكبر وتؤثر الدالة الحامضية للمحلول بصورة واضحة ومهمة على ملى النسبة المئويـة للامتزاز وان افضل دالة حامضية اكبر من (PH7) وان طبيعة الامتزاز فيزيائية وبعملية تلقائية تتحول الى كيميائية وعملية الامتزاز سريعة في بدايتها وان تطبيق مختلف النماذج الحركية والايزوثيرمية تم تم الحصول عليه وان طاقة التششيط الظاهرية بقيمتها واشارتها تدعم الدراسة الثرموداينيميكية . 


\section{(لمصادر}

1 .Dhantaj.T.M,Kariya.k.p,Bhave.N.S. "Physico Chemical Studies of Resin Derived from p-hydroxy benzoic acid .Diamin Benzoic with Formaldehyde and it's electrical conductivity study" Chemistry Journal vol.1.Issue 1,pp(1-8),(2011).

2.Yin.X.,w,Yao.z,Feng.R,Du.B,Yan.L,Wei.Q."Adsorption of benzoic acid from aqueous solution by three Kind of modified bentonites " J.Colloid.Intersci.,vol 359 ,pp(499-504).(2010).

3.Andreozzi.R,Marotta.R,Nicklas"Pharmacenticals in STP effluents and their photodegradation in aquatic environment"Chemosphere,vol.50,pp1319-1330,(2003).

4.Chor.k,Bocquet.J.F,Colbeam Justin.c,"comparative studies of phenol and salicylic acid photo catalytic degradation influence of adsorbed oxygen "mater.chem-plys.,vol 86.pp 123-131,(2004).

5.Yang.M,XU.A.H.DU,San.c,Li.c,"Removal of salicylic acid on perovslcite -type oxide $\mathrm{LaFeO}_{3}$ in catalytic wet air oxidation process" Hazard Mater J,vol.139 pp 8692,(2007).

6.Davor.K,Ivan,K,N,Nikola.k,"Adsorption of organic acids on metal oxides "The Umbrella Effect "Croation chemical Acta ,1139-1153 ,7(4).(1998)

7.Khaleel.I.Al-Niemi,Safwan.A.A,Thuka.A.,"Effect of using $\mathrm{MnO}_{2}$ As adsorbent substance on ionization constant and electrical conductivity for some aliphatic carboxylic acids contains hydroxyl group on $(\alpha)$ position, Al-Anbar university Journal for apure Science ,Vol(5).No(3),(2011)

8. Khaleel.I.Al-Niemi, Asmaa.M.A." Study the isothermic adsorption for some amino acids by using Manganize dioxide "The second scientific conference in chemistry university of Mosul , collage of science , chemistry Department.

9.Khaleel.I.Al-Niemi, Ibrahim.y.M," changing of electrical properties and adsorption mechanism for some dicarboxylic acids and factors affecting on it in presence of $\mathrm{MnO}_{2}$ as adsorbent substance" Journal of Tikrit university for apure science ,accepted for publication 18/6/2013

10. Khaleel.I.Al Niemi. Mohammed.M.H.Al-Niemi" Study the effecting of Manganize dioxide as adsorbent substance on electrical conductivity and degree of discossition for some amino acid " accepted for puplication in Journal of Tikrit university for apure science .(17/6/2012)

11 .Ho.Y.S,Mckoy.G"Application of kinetic Models to the sorption of copper II on 4he peat " Adsorption science \&Technology ,Vol .20 No.8,(2002). 
12 .Ali,A.s.s,serveh .s."Effects of sterilization and temperature on decrease Kinetic of phosphorus Bio Avaliability in two different soils types"J.soil.sci. plant Nutr .11(2):109-122,(2011).

13-Mohammed .N.I,Khalid.M.D,Marwan.z"Organic chemistry "Dar alketab for puplication Iraq,Mosul,university of Mosul (1988)

14. Yakout.S.M,Elsheriff .E,"Batch kinetics ,isotherm and thermodynamic studies of adsorotion of strontium from aqueous solution onto low cost rice -straw based Carbon "applied science Innovations pvt.Ltd,India Carbon -sci.Tech.1,144-153.(2010).

15.Baray.L.G,Dippy.J.F.,Hughes.S.R,Laxton.L.W,"chemical constitution and the dissociation constants of mono carboxylic acids. XVII,ortho-effects in substituted salicylic acids .J.Chem.Soc.,2404-8(1957).

16 . Pimental and Mecellean.G.C."The hydroqen Bond"Freeman w.H.san Francisco .(1960).

17.Hui.Q ,Ln,L.Bing.C"Critical review in adsorption Kinetic Models "Journal of univ.sci.A 10(5):716-724(2009).

18.Igwe.Jc,Abia.A.A"adsorption kinetics and intraparticle diffusivities for bio oremdiation of $\mathrm{Co}$ II ,Fe II , and $\mathrm{Cu}$ II ion s from Waste water using modified and unmodified maize Cob "International Journal of physical sciences vol 2(5) p.p 119126(2007).

19.Horsfan.M,A.I.S"Effects of temperature on the sorption of $\mathrm{pb}^{+2}$ from aqueous solution by caladium biocolor (Wild coccyaml bio mass"Electronic Journal of Technology vol 8,Num,2 pp 162-169(2005). 